\title{
Interaction of poly(lactic-co-glycolic acid) nanoparticles at fluid interfaces
}

\author{
Gergő Gyulai* and Éva Kiss
}

Laboratory of Interfaces and Nanostructures, Institute of Chemistry, Eötvös Loránd University, Budapest 112, PO Box 32, H-1518 Budapest, Hungary

*Corresponding author:

G. Gyulai

ggyulai@chem.elte.hu

\begin{abstract}
Hypothesis

Adsorption and localization of nanoparticles at fluid interfaces are key factors in processes like transport through membranes or emulsion stabilization. Adsorption of PLGA and Pluronic coated PLGA nanoparticles were studied at three different fluid interfaces. The effect of particle surface modification and type of interface was investigated with the aim of fine tuning interfacial interaction of the nanoparticles.

Experiments

Surface tension measurements were carried out to determine the surface activity and adsorption kinetics of the particles. Particles layers at the air/water interface were further studied using the Langmuir balance technique by recording the surface pressure -area isotherms. Interfacial rheological measurements were performed to characterize the structural properties of the nanoparticle interfacial films.

Findings

Interfacial adsorption and its kinetics were explained by the diffusion controlled adsorption theory and considering the energy barrier of particle transport to the interface. Surface modification by Pluronic increased the interfacial activity of nanoparticles at all interfaces. Surface activity of PLGA-Pluronic particles could be described by the contributions of both the PLGA NPs and the effective portion of their Pluronic shell. Both particle films present mainly elastic dilatational properties suggesting that particles are in kinetically separated state.
\end{abstract}

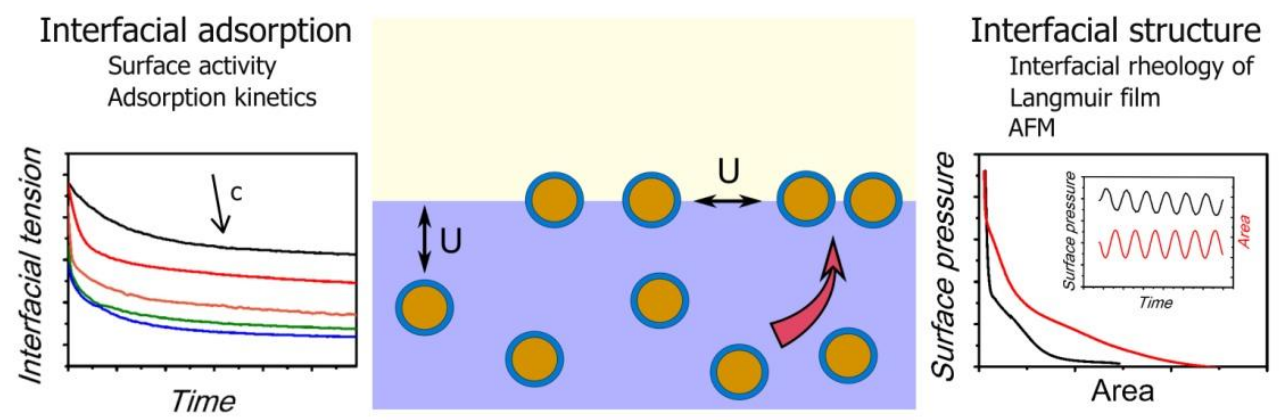

\section{Keywords}

PLGA nanoparticles

Pluronic stabilized nanoparticles

Adsorption kinetics

Interfacial dilatational rheology

Interfacial structure 


\section{Introduction}

Accumulation of nanoparticles at fluid or solid interfaces has great influence on their applicability in various fields of technology [1]. The self-assembly of particles at fluid interfaces have been investigated to create nanoporous membranes [2] and the fabrication of surface coatings with tuneable electrical, optical properties [3,4]. The adsorption layer of nanoparticles at fluid interfaces are used for the stabilization of emulsions and foams in the cosmetic, pharmaceutical and metallurgy fields $[5,6,7,8]$ as well as flotation of minerals [9]. Hydrophobic silica nanoparticles [10] and various types of polymeric microgels $[11,12,13]$ have shown great potential as stabilizers of Pickering emulsions. More recently biodegradable polymeric nanoparticles have been investigated as biocompatible alternatives for these materials to be used as stabilizer for medical emulsions [14].

Poly(lactic-co-glycolic acid) (PLGA) nano- and microparticles have been described as convenient platforms to carry drug molecules in the body $[15,16,17]$. Surface modification of these biodegradable particles with polyethylene oxide containing molecules has been shown to increase the biocompatibility of these systems $[18,19,20,21]$. Interfacial interactions play an essential role in drug delivery as drug carrier particles have to pass several interfaces in the body before they reach the site of action. The knowledge how these particle systems interact with various fluid and fluid-solid interfaces is crucial in the rational design of new therapeutic methods.

The behavior of particles at fluid interfaces was investigated in recent works to get information on the emulsion stabilizing properties. These studies mainly focus on the systems like soft microgels [11,22], micrometer scaled solid particles [23] or small inorganic nanoparticles [24,25].

In the present study biocompatible PLGA nanoparticles (NPs) in the size range of 100-200 nm designed for drug delivery were studied. A comparative investigation was carried out to analyze the effect of a polymeric surfactant surface modifier on the interfacial properties and interactions of the particles at three interfaces, air /water, n-octane/water and 1-octanol/water representing a series of interfaces between phases of different polarities. The above interfaces are considered as simple, well defined models of biointerfaces allowing the usage of physicochemical methods to follow the adsorption and interfacial interactions.

\section{Experimental}

\section{Materials}

Poly(DL-lactic-co-glycolic acid), PLGA with 50\% of lactic and 50\% of glycolic content (Mw: 5000075000), was obtained from Sigma-Aldrich, Hungary. Poly(ethylene oxide)/poly(propylene oxide)/poly(ethylene oxide), PEO-PPO-PEO triblock copolymer, Pluronic F127 ( $\mathrm{M}_{\mathrm{w}}$ : 12700) (provided by BASF Hungaria Kft.), was applied as received. The composition of the Pluronic 127 is on average $202 \mathrm{EO}$ units and $56 \mathrm{PO}$ units according to the manufacturer.

All organic solvent were of analytical grade. Acetone was used in the preparation of nanoparticles. Dichloromethane was used for cleaning the Langmuir trough and its barriers. Aqueous sols of NPs were mixed with 2-propanol for the spreading on the aqueous surface. n-octane and 1-octanol were equilibrated with water for one day prior interfacial tension measurements.

Doubly distilled water checked by its conductivity $(<5 \mathrm{mS})$ and surface tension $(>72.0 \mathrm{mN} / \mathrm{m}$ at $25 \pm 0.5^{\circ} \mathrm{C}$ ) was used as medium for aqueous solutions and as the subphase in the Langmuir balance experiments.

\section{Preparation and characterization of PLGA nanoparticles}

PLGA nanoparticles with a narrow size distribution were prepared using the nanoprecipitation method as described previously [26,27]. Briefly PLGA was dissolved in acetone at a concentration of $10 \mathrm{~g} / \mathrm{L}$. $5 \mathrm{~mL}$ of the organic solution was added dropwise to either $50 \mathrm{~mL}$ of pure water or $50 \mathrm{~mL}$ of $1 \mathrm{~g} / \mathrm{L}$ Pluronic F127 aqueous solution under magnetic stirring $(500 \mathrm{rpm})$ to create pure PLGA or Pluronic 
stabilized PLGA NPs. After the complete evaporation of acetone PLGA sol was centrifuged at $3500 \mathrm{~g}$ for $10 \mathrm{~min}$ to remove possible particle aggregates. The supernatant, containing the nanoparticles, was separated and concentrated to a final volume of $5 \mathrm{~mL}$ by membrane filtering. Pluronic stabilized NPs were further purified by centrifugation at $12000 \mathrm{~g}$ for $20 \mathrm{~min}$. The supernatant was removed and the pellet containing the particles was redispersed in bidistilled water. This purification procedure was repeated 4 times, when the surface tension of the supernatant was $>72 \mathrm{mN} / \mathrm{m}$. The mass concentration of the sols was determined by dry mass measurement.

Average hydrodynamic size and polydispersity of the PLGA NPs were determined using a dynamic light scattering (DLS) system (Brookhaven Instruments, USA) consisting of a BI-200SM goniometer and a BI-9000AT digital autocorrelator. As a light source a Coherent Genesis MX488-1000STM laserdiode system operating at $488 \mathrm{~nm}$ wavelength and emitting vertically polarized light was used. Measurements were carried out at a detection angle of $90^{\circ}$ and a temperature of $25{ }^{\circ} \mathrm{C}$ with nanoparticle sample appropriately diluted with doubly distilled water. The recorded autocorrelation functions were analyzed by the second order cumulant expansion and Contin method.

\section{Zeta potential measurement}

The determination of electrophoretic mobility of nanoparticles was carried out by means of Malvern Zetasizer Nano $\mathrm{Z}$ apparatus at $25{ }^{\circ} \mathrm{C}$. For calculations the zeta potential of octane/water and octanol/water emulsions were also determined. Oil droplets were formed according to the method described by Marinova et al [28]. The oil and water were added to a glass container that was heated to $60{ }^{\circ} \mathrm{C}$ for $1 \mathrm{~h}$ while the solvents were stirred gently. Samples were taken from the aqueous phase that became cloudy upon cooling to $25{ }^{\circ} \mathrm{C}$ indicating the formation of oil droplets from the oversaturated solution. Measurements were carried out immediately. Smoluchowski approximation was used to calculate zeta potential $(\zeta)$ from mobility values. Results were collected in triplicates, the $\zeta$ values were reproducible to $\pm 1 \mathrm{mV}$.

\section{Surface activity of nanoparticles}

Adsorption of nanoparticles at fluid interfaces was monitored through the measurement of surface/interfacial tension [29]. Axisymmetric drop shape analysis of droplets of the aqueous sols was carried out in an OCA15+ (Dataphysics, Germany) optical contour analysis instrument. $10 \mu \mathrm{L}$ droplets were created in air, octane or 1-octanol outer phases, at $25{ }^{\circ} \mathrm{C}$. The octane and octanol media were presaturated with water. Surface and interfacial tension values were followed for $1 \mathrm{~h}$ at various sol concentrations in the range of $0.25-4 \mathrm{~g} / \mathrm{L}$. The measurement time was limited to $1 \mathrm{~h}$ to allow the particles to approach their equilibrium surface tension but decrease the risk of possible trace contaminants influencing the measured values. Three parallel measurements were carried out for all systems and surface tension values were reproducible to $\pm 0.5 \mathrm{mN} / \mathrm{m}$.

\section{Langmuir monolayer studies}

Monolayer studies of the NPs were performed in a Langmuir through, KSV MiniMicro $(5 \times 20 \times 0.6$ $\mathrm{cm}^{3}$ ) with two moving barriers to provide symmetric film compression with maximum compression ratio of 5.2. The surface pressure, the difference in surface tension between the pure interface and the particle covered interface $\left(\Pi=\gamma_{0}-\gamma\right)$, was recorded with an accuracy of $0.05 \mathrm{mN} / \mathrm{m}$ with the aid of a Wilhelmy plate made of chromatography paper (Whatman Chr1) connected to a force transducer. The trough and barriers were made of Teflon and cleaned carefully with dichloromethane and water. The trough was placed on a thermo-regulated plate and into a box of plexiglass in order to minimize air turbulence and possible contamination [30]. All measurements were performed at $(25 \pm 0.5){ }^{\circ} \mathrm{C}$. Double distilled water was used as the subphase. 
For the preparation of Langmuir layers the aqueous particle sol was mixed with 2-propanol at 1:1 volume ratio. No aggregation was detectable by visual observation and DLS measurements. Known amount of the sample was spread on the water subphase and the particles were left to equilibrate and the 2-propanol to evaporate for $30 \mathrm{~min}$. Surface pressure - area isotherms were determined at barrier speed of $32 \mathrm{~mm} / \mathrm{min}$. Isotherms were recorded in triplicates and with varying spreaded NP amounts. Measurements were highly reproducible allowing for the construction of composite overlapping isotherms.

\section{Interfacial rheology studies}

Interfacial rheological properties of the particle films were investigated at all interfaces by axysimmetric drop shape analysis. For low amplitude area perturbations the absolute value of the complex dilatational modulus $(|E|)$ can be calculated as

$|E|=\frac{\Delta \Pi}{\Delta A / A_{0}}$,

where $\Delta \Pi$ and $\Delta A$ are the changes in surface pressure and area, while $A_{0}$ is the initial surface area. $|E|$ was determined at fixed surface pressures close to the equilibrium surface pressures of the systems after $1 \mathrm{~h}$ particle adsorption time. The surface of the sol droplets was compressed by reducing their volume at a constant rate of $0.05 \mu \mathrm{L} / \mathrm{s}$. Dilatational modulus was calculated according to Eq. 1 at a maximum $10 \%$ relative surface area compression.

For the air/water interface the Langmuir balance technique allows for the systematic investigation of the dilatational modulus at any given surface pressure. For this oscillating barrier measurements were carried out on Langmuir monolayer of NPs at increasing layer compression states. The complex dilatational modulus and its elastic and viscous components could be determined from these measurements.

The films were compressed to the desired surface pressure value at $32 \mathrm{~mm} / \mathrm{min}$ speed. Symmetric oscillating movement of the barriers was then initiated at $0.05 \mathrm{~Hz}$ frequency and constant relative area oscillation amplitude $( \pm 2 \%)$. Fourier transformation was used to determine the amplitude and phase angle of the area and surface pressure oscillation. For low amplitude area perturbations the complex dilatational modulus can be written as

$$
E=E^{\prime}+i E^{\prime \prime}=|E| e^{i \Phi}=|E|(\cos \Phi+i \sin \Phi) \text {, }
$$

where $E^{\prime}$ is the elastic and $E^{\prime \prime}$ is the viscous component of the modulus and $\Phi$ is the phase angle between the surface area and surface pressure oscillations [31]. $|E|$ was calculated according to Eq. 1. from the amplitudes of the area and surface pressure oscillation.

\section{Morphology of NP monolayers}

The morphology of the Langmuir monolayers of the PLGA NPs was investigated by atomic force microscopy (AFM). Langmuir-Schaeffer technique was employed to transfer the surface film of particles onto a solid support in the Langmuir balance. Microscope cover glasses $\left(22 \times 40 \times 0.16 \mathrm{~mm}^{3}\right)$ were used as substrates that were previously cleaned in freshly prepared persulfuric acid solution followed by gas phase functionalization using (3-aminopropyl) triethoxysilane (APTES).The resulting amine functionalized surface promoted the immobilization of the particles and inhibited the reorganization of the film during drying. The transferred films were imaged in air at room temperature using a XE-100 atomic force microscope (AFM) system (Psia, South Korea). Measurements were carried out in noncontact mode using NSC15 (Micromash, Estonia) cantilevers. Images were collected at 20-30 randomly selected locations with typical scan area of $20 \times 20 \mu \mathrm{m}^{2}$. 


\section{Wettability of PLGA and PLGA-Pluronic surfaces}

Flat surfaces as model of the NPs were prepared using the solvent casting method. PLGA and PLGAPluronic mixtures (2\% Pluronic F127) were dissolved in dichloromethane to a concentration of $10 \mathrm{~g} / \mathrm{L}$. Glass microscope slides $\left(2 \times 2 \mathrm{~cm}^{2}\right)$ were used as substrates for the films that were previously cleaned in freshly prepared persulfuric acid solution. The substrates were covered by the polymer solutions $(200 \mu \mathrm{L})$ and the solvent was left to slowly evaporate under cover. Solvent residues were removed by heating the samples to $60{ }^{\circ} \mathrm{C}$ above the glass transition temperature of the polymers. This also promotes the accumulation of Pluronic in the upper layers of the polymer mixture as was shown previously [19,32]. Prior to contact angle measurements the samples were equilibrated in water for $1 \mathrm{~h}$ to approximate the state of the polymers to those on the nanoparticle sols. Measurements were carried out in water using the captive bubble/droplet method (Fig. 1), with air, n-octane and 1-octanol as test materials. Small droplets of the test materials were deposited to the polymer surface. Images of the droplets/bubbles were recorded using OCA15+ (Dataphysics, Germany) optical contour analysis instrument following repeated increase and decrease in the droplet volume. In the captive bubble/droplet method increasing the droplet volume enables the measurement of the receding water contact angle, while decreasing the volume leads to the detection of the advancing contact angle. For each system 20 measurements were carried out. Advancing and receding contact angles $\left(\theta_{\mathrm{A}}, \theta_{\mathrm{R}}\right)$ were determined using the Laplace-Young method and the equilibrium contact angle $(\theta)$ was estimated as their cosine average: $\cos \theta=\left(\cos \theta_{\mathrm{A}}+\cos \theta_{\mathrm{R}}\right) / 2$.

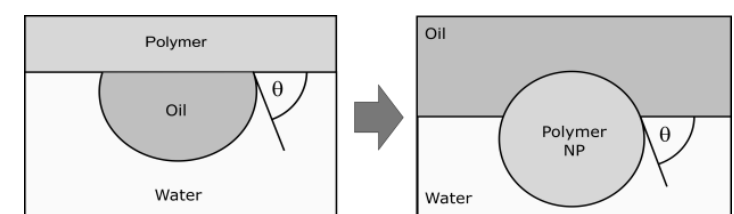

Figure 1. Experimental setup of the captive bubble/droplet contact angle measurement and its relation to the particle contact angle at the interface

\section{Results and discussion}

\section{Size and zeta potential of PLGA NPs}

Size of pure PLGA and Pluronic F127 stabilized PLGA nanoparticles was determined by DLS measurements. The particles had a number average hydrodynamic diameter of $90 \mathrm{~nm}$ for the pure PLGA and $98 \mathrm{~nm}$ for the Pluronic stabilized NPs. Polydispersity indices were below 0.1 (0.08 and 0.06 respectively) indicating a narrow size distribution. The size and shape of the particles was also characterized by AFM. 2000-2500 particle height profiles were used in the evaluation. Particles were found to be unimodal in size distribution and had average diameters of $85 \mathrm{~nm}$ and $92 \mathrm{~nm}$ for PLGA and PLGA-Pluronic NPs, respectively. In the model calculations the particle diameters determined by AFM were used.

Electrophoretic mobility studies revealed that PLGA and PLGA-Pluronic NPs had negative zeta potentials of -40 and $-16 \mathrm{mV}$, respectively. This charge character is the result of the presence of surface carboxylic polymer chain end groups of PLGA. In the case of PLGA-Pluronic particles the zeta potential is reduced due to the screening effect of the Pluronic surface layer.

\section{Surface activity of NPs at fluid interfaces}

Interfacial adsorption properties of the polymer NPs were investigated using axisymmetric drop shape analysis. Measurements were carried out at the air/water, n-octane/water and 1-octanol/water 
interfaces at various NP concentrations $(0.25-4.00 \mathrm{~g} / \mathrm{L})$. Example adsorption curves are presented in Fig 2.
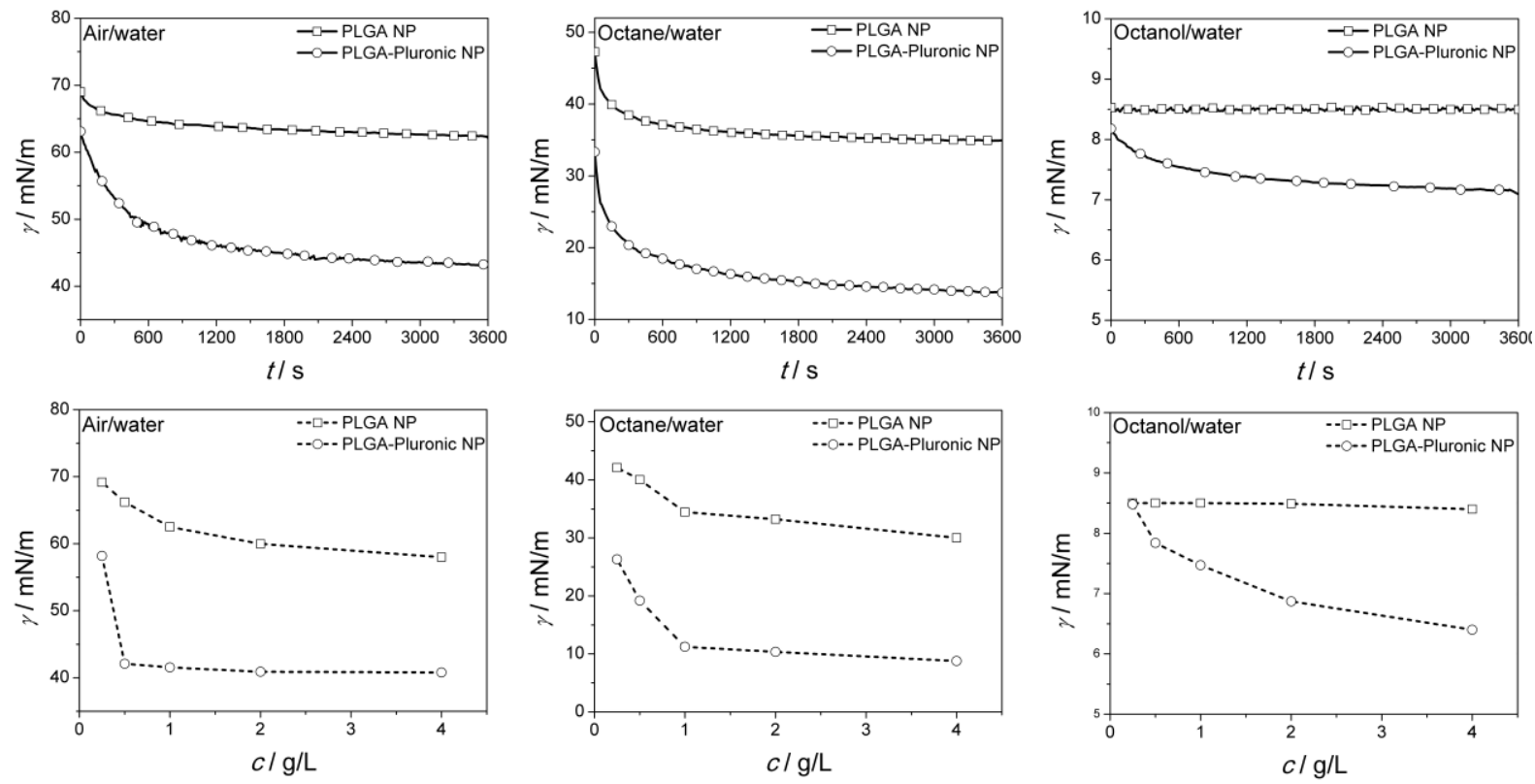

Figure 2. Surface and interfacial tension of PLGA and Pluronic modified PLGA NPs as a function of time at concentration of $2 \mathrm{~g} / \mathrm{L}$, and equilibrium surface and interfacial tension values as a function of initial bulk concentration

Following the adsorption of the particles it was found that primarily at lower concentration no equilibrium surface tension was reached over the $1 \mathrm{~h}$ measurement time. Assuming a diffusion controlled adsorption the equilibrium surface tension can be determined. In such a case an asymptotic approximate solution of the Ward and Tordai equation for $t \rightarrow \infty$ case gives

$\gamma_{t \rightarrow \infty}=\gamma_{\infty}-\frac{R T \Gamma^{2}}{2 c_{0}}\left(\frac{\pi}{D}\right)^{1 / 2} t^{-1 / 2}$

where $\gamma$ and $\gamma_{\infty}$ are the dynamic and equilibrium surface tensions, $R$ is the universal gas constant $T$ is the temperature, $\Gamma$ is the surface excess concentration, $c_{0}$ is the bulk concentration and $D$ is the diffusion coefficient [33]. The extrapolated surface tension values as a function of initial bulk particle concentration are presented in Fig 2. Surface tension values appear to converge to a limiting minimum value at higher concentrations. These $\gamma_{\mathrm{eq}}$ are presented as surface pressure data $\left(\Pi_{\mathrm{eq}}=\gamma_{0}-\gamma_{\mathrm{eq}}\right)$ in Table 1. The surface tension of a nanoparticulate system is related to the surface coverage of the interface by the particles. The limiting value corresponds to a maximum equilibrium packing of the nanoparticles at the interface determined by the size of the NPs and their interparticle interactions.

Table 1. Equilibrium surface pressure $\left(\Pi_{\mathrm{eq}}\right)$ and dilatational modulus $(|E|)$ of nanoparticle layer at different interfaces

\begin{tabular}{|c|c|c|c|c|c|}
\hline \multirow[t]{2}{*}{ Interface } & \multirow{2}{*}{$\begin{array}{c}\text { Pure interface } \\
\gamma_{0} / \mathrm{mN} / \mathrm{m}\end{array}$} & \multicolumn{2}{|c|}{ PLGA NP } & \multicolumn{2}{|c|}{ PLGA-Pluronic NP } \\
\hline & & $\Pi_{\mathrm{eq}} / \mathrm{mN} / \mathrm{m}$ & $|E| / \mathrm{mN} / \mathrm{m}$ & $\Pi_{\mathrm{eq}} / \mathrm{mN} / \mathrm{m}$ & $|E| / \mathrm{mN} / \mathrm{m}$ \\
\hline air/water & 72.0 & 16.0 & 9.8 & 31.2 & 10.0 \\
\hline n-octane/water & 50.0 & 20.2 & 10.6 & 41.0 & 14.6 \\
\hline 1-octanol/water & 8.5 & 0.1 & 0.1 & 2.1 & 1.7 \\
\hline
\end{tabular}


PLGA and PLGA-Pluronic particle systems reduced the surface tension of water and the octane/water interfacial tension as well. On the contrary, no measureable change could be observed at the octanol/water interface for pure PLGA NPs. The Pluronic covered PLGA NPs however exhibited surface activity in the case of all three interfaces.

Considering the degree of surface/interfacial tension change caused by the presence of NPs, the Pluronic modification enhances greatly the interfacial activity of the nanoparticles at all interfaces.

\section{Interfacial rheology}

Interfacial rheological measurements were also carried out in drop shape analysis measurements, where a droplet of the aqueous sol of particles was analyzed after $1 \mathrm{~h}$ adsorption time. The determined absolute values of the interfacial dilatational moduli close to the equilibrium surface tension are presented in Table 1.

Measurements carried out with sols of varying particle concentrations showed little variation in the determined value of $|E|$. The obtained dilatational moduli for air/water and octane/water interfaces were found to be relatively small values, $8-11 \mathrm{mN} / \mathrm{m}$ and $10-20 \mathrm{mN} / \mathrm{m}$ for PLGA and Pluronic modified PLGA NPs respectively, indicating that the interfacial films are highly compressible and the particles are well separated up to their equilibrium surface pressure state. The determined moduli values appear to be similar to those found by Alvarez et al. for charged polymeric particles at their equilibrium surface concentrations [34]. On the octanol/water interface the determined moduli are close to zero indicating the low coverage of the interface by the particles.

\section{Langmuir monolayer studies}

The Langmuir balance technique offers a more in-depth study of the particle layer at the air/water interface. Pluronic stabilized and pure PLGA NPs were spread at surface of aqueous subphase in a Langmuir balance to form monoparticular layers. Surface pressure - area isotherms were acquired by compression of the particle layer (Fig. 3). As a comparison, isotherm of the molecular layer of Pluronic F127 was also recorded.

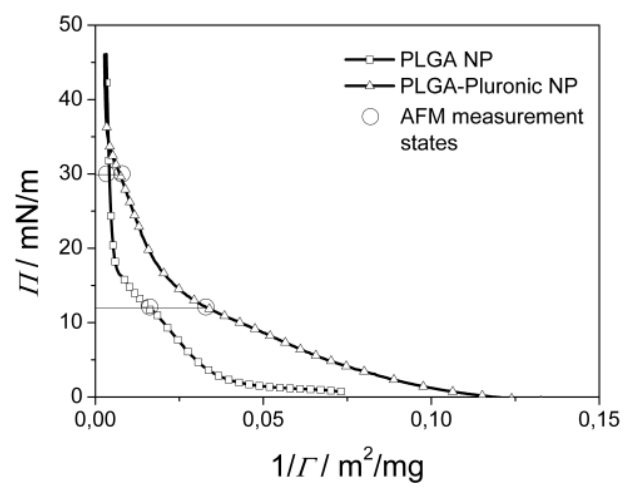

Figure 3. Surface pressure - area isotherm of PLGA and PLGA-Pluronic NPs at the air/water interface

In the case of pure PLGA NPs the isotherm can be split into three different regions. The low surface concentration region where the surface pressure is close to zero corresponds to a gas analogous state with negligible interparticle interactions. This is followed by a steady increase in surface pressure up till $16 \mathrm{mN} / \mathrm{m}$ where the particles are getting closer and start to interact with each other. Above $16 \mathrm{mN} / \mathrm{m}$ with further surface area reduction a steep increase in surface pressure is observed. This means the particles are approaching their maximum packing density. 
Pluronic stabilized particle films exhibit a significantly different isotherm. Already at low surface concentrations a prolonged gradual increase in surface pressure is observed. Above $15 \mathrm{mN} / \mathrm{m}$ the surface pressure increase becomes steeper. Finally from $33 \mathrm{mN} / \mathrm{m}$ the surface pressure increase becomes steep alike that of PLGA NPs indicating the formation of a particle film with maximum packing. The isotherms were highly reproducible for both particle systems and no notable shift was detectable in consecutive compression-expansion cycles. No signs of a layer collapse could be detected in either case up till the maximum investigated surface pressure of $45 \mathrm{mN} / \mathrm{m}$.

Structural properties of the nanoparticle layer were investigated by oscillating the area of the film. The dilatational moduli of the Langmuir films at increasing surface pressure were determined according to Eq.1-2 and are presented in Fig 4.

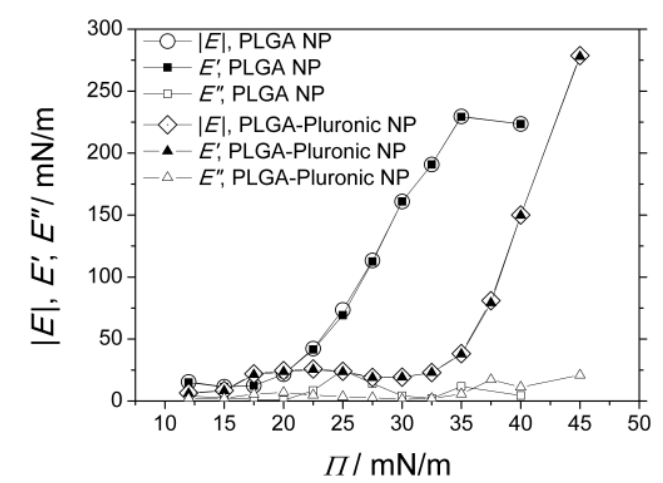

Figure 4. Dilatational modulus $(|E|)$ and its components $\left(E^{\prime}, E^{\prime \prime}\right)$ of PLGA and PLGA-Pluronic NPs at the air/water interface as a function of surface pressure

The $|E|$ values determined by the oscillating barrier method at 15 and $30 \mathrm{mN} / \mathrm{m}$ for PLGA and PLGAPluronic NPs correspond well to the values determined by the droplet compression technique close to the equilibrium surface pressure. This supports the assumption that Langmuir layers and adsorption layers of the particles are in a similar state.

Pure PLGA particle layers exhibit a relatively small dilatational modulus of $15 \mathrm{mN} / \mathrm{m}$ that appears to change only slightly up till the surface pressure of $20 \mathrm{mN} / \mathrm{m}$. The film deformation appears to be mainly elastic in nature, indicated by the small contribution of the imaginary part of the complex modulus, $E^{\prime \prime}$, describing the viscous property. This behavior might be the consequence of long-range repulsive forces between the particles [23]. Above surface pressures $20 \mathrm{mN} / \mathrm{m}$ a change in this behavior is observed, where the real part of the dilatational modulus $\left(E^{\prime}\right)$ increases to $250 \mathrm{mN} / \mathrm{m}$ while imaginary part remains an insignificant contributor. These data correspond to the formation of continuous structure of closely packed particles.

Pluronic stabilized nanoparticles exhibit a different behavior. At low surface pressures, below $17 \mathrm{mN} / \mathrm{m}$ the dilatational modulus is small, around $7 \mathrm{mN} / \mathrm{m}$ and mostly elastic in nature. This value is markedly smaller than the one obtained for pure PLGA particles which can be explained by lower surface concentration of PLGA-Pluronic NPs in this surface pressure region. Between surface pressure of 17 and $33 \mathrm{mN} / \mathrm{m}$ a stepwise increase in the dilatational modulus of the film was observed that remained at a relatively constant value of $22 \mathrm{mN} / \mathrm{m}$. This increased modulus coincides with a break in the surface pressure isotherm of the system and could be the result of increased long range repulsion between the particles, presumably the sum of electrostatic interactions and the polymer-polymer steric repulsion due to the presence of the Pluronic surface layer. This assumption is strengthened by the fact that molecular layer of Pluronic F127 exhibits a similar dilatational modulus ( $18 \mathrm{mN} / \mathrm{m})$ in this surface pressure region. Further compressing the particles above $33 \mathrm{mN} / \mathrm{m}$, along with the steep slope of the 
isotherm, a sharp increase could be detected in the dilatation modulus as well signifying the formation of a close packed structure.

\section{Morphology of NP surface layers}

To visualize the structure of the particle layer the Langmuir films were transferred to a solid support using the Langmuir-Schaeffer technique and analyzed by AFM (Fig. 5). Films were immobilized at two surface pressures, 12 and $30 \mathrm{mN} / \mathrm{m}$.
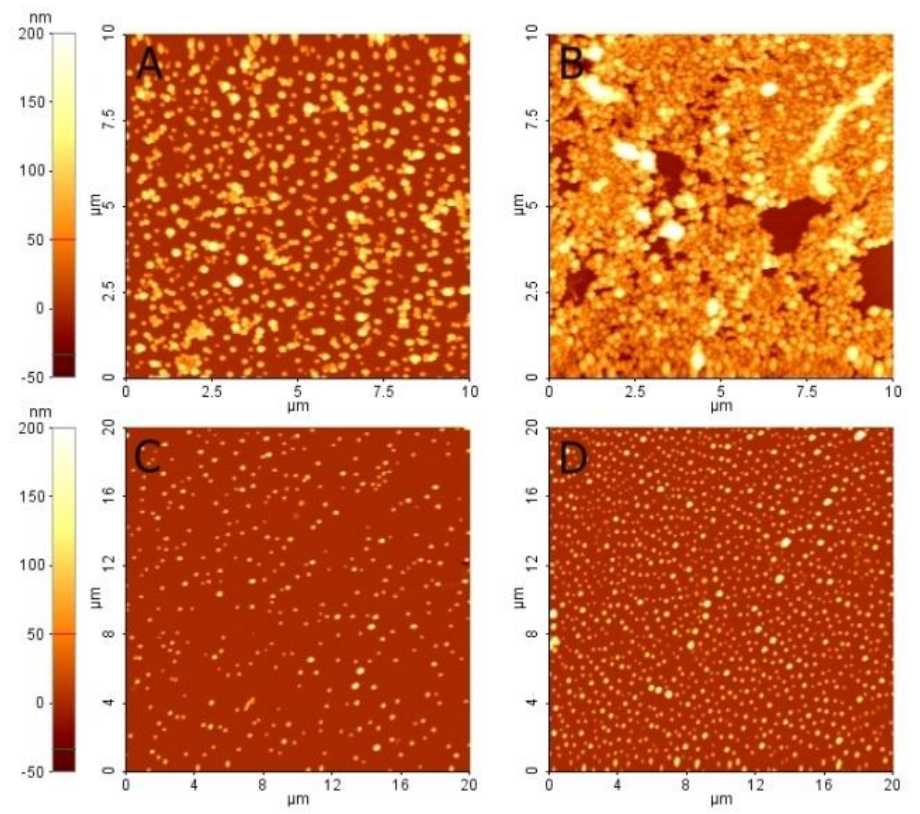

Figure 5. AFM image of PLGA and PLGA-Pluronic NP layers transferred at $12 \mathrm{mN} / \mathrm{m}$ (A: PLGA, C: PLGA-Pluronic) and $30 \mathrm{mN} / \mathrm{m}$ (B: PLGA, D: PLGA-Pluronic)

In the case of pure PLGA NPs already at $12 \mathrm{mN} / \mathrm{m}$ a relatively dense layer was formed where a small number of particles stick together to form loose aggregates. Increasing the compression further the particles become closely packed and the layer start to collapse leading to the formation of particle multilayers. It should be noted that the buckling of the particle layers appear to be reversible as the compression and expansion parts of the Langmuir isotherms of the films appeared to be highly reproducible. The Langmuir-Schaeffer films were always prepared after recording three consecutive isotherms where the particles were subjected to high surface pressures leading to the formation of structures seen in Fig 5B. On the other hand at lower surface pressures apart from the presence of small aggregates individual particles are also present as seen in Fig 5A. This indicates that the continuous tightly packed particle islands break up when no longer subjected to high compression. These findings correspond well with those of Razavi et al [35]. The experimental observations of reproducible surface pressure isotherms with elastic deformations point to a reversible folding. The wettability of the PLGA NPs is also close to the range where a buckling film relaxation instead of particle expulsion can be expected to be the main mode of film relaxation.

With the Pluronic modified layers no aggregation was detected. Even at $30 \mathrm{mN} / \mathrm{m}$ surface pressure the particles remained well separated and no signs of even loose aggregates could be found. Apart from the electrostatic repulsion between the particles, the steric barrier of the Pluronic surface layer provides a further driving force against aggregation, indicating high kinetic stability for the system.

Since the particles have a slightly increased wettability compared to pure PLGA particle expulsion could be expected. On the contrary neither expulsion nor film buckling was observed directly. When the reproducibility of the isotherms and the elastic property of the film were considered it is reasonable to assume that at sufficiently high surface pressures reversible folding can occur. 


\section{Free energy of interfacial accumulation of NPs}

To understand the interfacial adsorption of the PLGA and PLGA-Pluronic NPs further analysis of the obtained data was carried out. Nanoparticles are widely assumed to adsorb to fluid interfaces in an irreversible manner. The irreversible nature of adsorption is a fair assumption considering the free energy change occurring when a nanometer sized object appears at the interface [36]:

$$
\Delta G=-r^{2} \pi \gamma_{\text {ow }}(1-|\cos \theta|)^{2}
$$

where $r$ is the radius of the particles $\gamma_{\text {ow }}$ is the interfacial tension of the particle free interface and $\theta$ is the contact angle at the particle surface. For particles with a diameter $>10 \mathrm{~nm}$ and with contact angle notably different from $0^{\circ}$ or $180^{\circ}$, the free energy of their desorption $(-\Delta G)$ would significantly exceed their thermal energy, $3 / 2 k_{\mathrm{B}} T$, thereby their adsorption could be considered irreversible.

Eq. 4 has been used to calculate $\Delta G$ for the particles. The determined energy values along with the measured contact angle used in the calculations are presented in Table 2.

Table 2. Contact angle $(\theta)$, calculated free energy of adsorption $(\Delta G)$, Stokes-Einstein $\left(D_{0}\right)$ and effective $\left(D_{\text {eff }}\right)$ diffusion coefficient of NPs at different interfaces

\begin{tabular}{llcccc}
\hline Particle & Interface & $\theta /{ }^{\circ}$ & $\Delta G / k_{\mathrm{B}} T$ & $D_{0} / \mathrm{m}^{2} / \mathrm{s}$ & $D_{\text {eff }} / \mathrm{m}^{2} / \mathrm{s}$ \\
\hline PLGA & air/water & 74 & $-5.2 \times 10^{4}$ & $5.8 \times 10^{-12}$ & $1.0 \times 10^{-14}$ \\
PLGA-Pluronic & air/water & 65 & $-3.9 \times 10^{4}$ & $5.3 \times 10^{-12}$ & $4.0 \times 10^{-14}$ \\
PLGA & n-octane/water & 100 & $-4.7 \times 10^{4}$ & $5.8 \times 10^{-12}$ & $2.2 \times 10^{-14}$ \\
PLGA-Pluronic & n-octane/water & 106 & $-4.2 \times 10^{4}$ & $5.3 \times 10^{-12}$ & $1.6 \times 10^{-13}$ \\
PLGA & 1-octanol/water & 163 & $-2.1 \times 10^{1}$ & $5.8 \times 10^{-12}$ & $2.3 \times 10^{-14}$ \\
PLGA-Pluronic & 1-octanol/water & 143 & $-5.6 \times 10^{2}$ & $5.3 \times 10^{-12}$ & $7.4 \times 10^{-14}$ \\
\hline
\end{tabular}

*Note: Contact angle values were reproducible to $\pm 1^{\circ}$ leading to an error of $\pm 10 \%$ in the calculated $\Delta G$ values

The adsorption is a thermodynamically favorable process for both types of NPs at all interfaces and the energy of desorption, $-\Delta G$ significantly exceeds the thermal energy available to the particles. For the air/water and octane/water interfaces a large energy profit $4 \times 10^{4}-5 \times 10^{4} k_{\mathrm{B}} T$ is present corresponding to their high surface activity. At the octanol/water interface however the energy decrease is much smaller (only $20 k_{\mathrm{B}} T$ for pure PLGA and $560 k_{\mathrm{B}} T$ for the PLGA-Pluronic NPs), that could be the reason for the major reduction in the measured surface activity (Table 1).

\section{Kinetics of NP adsorption}

Although the adsorption is thermodynamically favored, adsorption kinetics of the particles depends on multiple parameters. The kinetics of adsorption can be modeled using the Ward-Tordai theory [37]. Here the adsorption process is assumed to be purely diffusion controlled, meaning the rate of adsorption depends only on the rate of particles arriving at the interface. The binding to the interface is considered to be instantaneous compared to the rate of diffusion. This way in the absence of an energy barrier at the interface the adsorption process should be only dependent on the particle diffusivity and concentration. According to Bizmark et al. [38] the initial decay of surface tension can be given as a modified version of the Ward-Tordai theory as

$\gamma=\gamma_{0}-2 N_{A} c_{0} \Delta G\left(\frac{D_{\text {eff }} t}{\pi}\right)^{1 / 2}$

where $\gamma$ is the interfacial tension at time $t, \gamma_{0}$ is the interfacial tension of the pure interface, $N_{\mathrm{A}}$ is the Avogadro number, $c_{0}$ is the bulk concentration of the particles, $\Delta G$ is the adsorption free energy (Table 2) of the particles and $D_{\text {eff }}$ is the apparent diffusion coefficient. If the adsorption follows a diffusion 
controlled transport then changes in interfacial tension as a function of $t^{1 / 2}$ should show a linear relationship.

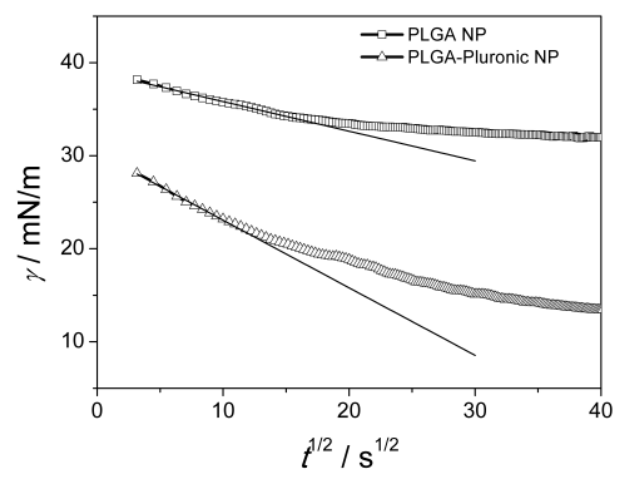

Figure 6. Interfacial tension of nanoparticles at the octane/water interface as a function of the square root of time

As can be seen in the Fig. 6 the initial parts of the adsorption show a linear tendency indicating that particle adsorption is indeed diffusion controlled up to a point where a significant portion of the surface becomes covered by the particles. The effective diffusion coefficient was calculated according to Eq. 5 from the slope of the initial parts of the $\gamma$ vs $t^{1 / 2}$ plots. The determined values are collected in Table 2.

The diffusion coefficient of the particles in bulk medium $\left(D_{0}\right)$ can also be calculated using the StokesEinstein equation,

$D_{0}=\frac{k_{\mathrm{B}} T}{6 r \pi \eta}$

where $k_{\mathrm{B}}$ is the Boltzmann constant, $T$ is the temperature, $r$ is the particle radius and $\eta$ is the viscosity of the medium. Comparing the two diffusion coefficients $D_{\text {eff }}$ appears to be considerably smaller than $D_{0}$.

This discrepancy can be interpreted with the existence of an energy barrier hindering the adsorption of the particles. The total interaction between a particle and an interface can be written according to the DLVO theory as

$U_{\text {tot }}=U_{\mathrm{vdW}}+U_{\mathrm{EDL}}+U_{\text {image }}$

where $U_{\mathrm{vdW}}$ is the van der Waals interaction, $U_{\mathrm{EDL}}$ is the electric double layer interaction between the particle and the interface and $U_{\text {image }}$ is the particle-image charge interaction [24]. The interactions can be modeled as an interaction between a sphere and a flat plate. In this geometry the van der Waals interaction can be written as

$U_{\mathrm{vdW}}=-\frac{A_{132}}{6}\left[\frac{r}{h}+\frac{r}{h+2 r}+\ln \left(\frac{h}{h+2 r}\right)\right]$

where $r$ is the particle radius, $h$ is the separation distance between the surfaces and $A_{123}$ is the effective Hamaker constant [39]. The Hamaker constant, where a particle (1) interacts with a bulk phase (2) over a medium (3), can be approximated from the vacuum Hamaker constants of the pure materials as $A_{132}=\left(\sqrt{A_{11}}-\sqrt{A_{33}}\right)\left(\sqrt{A_{22}}-\sqrt{A_{33}}\right)$

For the calculation the Hamaker constant of PLGA, $A_{11}$ was considered $6.5 \times 10^{-20} \mathrm{~J}$ [40] and $3.7 \times 10^{-20} \mathrm{~J}$ for water $\left(A_{33}\right)$ [41]. For $A_{22}, 0,4.5 \times 10^{-20}$ and $7.3 \times 10^{-20} \mathrm{~J}$ was used for air, n-octane [41] and 1-octanol respectively. The Hamaker constant of octanol was estimated using the $A=(4 \pi / 1.2) \gamma^{\mathrm{d}} a_{0}$ relation [42], where the dispersion component of the surface tension, $\gamma^{\mathrm{d}}$ is $27.5 \mathrm{mN} / \mathrm{m}$ while the equilibrium molecular spacing of octanol, $a_{0}$ is and $0.56 \mathrm{~nm}$ [43]. This means that the van der Waals 
interaction between PLGA and the fluid interface is attractive in nature in the case of octane and octanol, while it is repulsive for the air/water interface.

The image charge interaction arises from the difference in dielectric constants of the fluids over the interfaces. Near a flat interface, the force on a charge $q$ can be handled as an electrostatic interaction with an "image charge" $\left(q_{\mathrm{im}}\right)$ located in the position of a mirror image across the interface. The image charge can be calculated as

$q_{\mathrm{im}}=q \frac{\varepsilon_{1}-\varepsilon_{2}}{\varepsilon_{1}+\varepsilon_{2}}$

where $\varepsilon_{1}$ and $\varepsilon_{2}$ are the dielectric constants of the charge containing medium (1) and the charge-free medium (2) [44]. In the case where the particles are in a high dielectric medium, like water and the contacting phase is a low dielectric medium the image charge will have the same sign, therefore the image charge interaction will be repulsive in nature. The image charge surface potential was calculated by the Grahame equation as [45]

$$
\Psi_{\mathrm{im}}=\frac{2 k_{\mathrm{B}} T}{z e} \operatorname{arcsinh}\left[\frac{\varepsilon_{1}-\varepsilon_{2}}{\varepsilon_{1}+\varepsilon_{2}} \sinh \left(\frac{z e \Psi_{\mathrm{NP}}}{2 k_{\mathrm{B}} T}\right)\right]
$$

The image charge interaction (Eq. 12) and electric double layer interaction (Eq. 13) between the particle and the interface was calculated based on the linear superposition method (LSA) proposed by Lin and Wiesner [46]. The model describes the interaction between a charged sphere and a charged infinite plate:

$$
\begin{aligned}
& U_{\text {image }}=32 \pi \varepsilon r\left(\frac{k_{\mathrm{B}} T}{z e}\right)^{2} \tanh \left(\frac{z e \Psi_{\mathrm{NP}}}{4 k_{\mathrm{B}} T}\right) \tanh \left(\frac{z e \Psi_{\mathrm{im}}}{4 k_{\mathrm{B}} T}\right)\left[e^{-2 \kappa h}\right] \\
& U_{\mathrm{EDL}}=\frac{64 \pi \varepsilon}{\kappa}\left(\frac{k_{\mathrm{B}} T}{z e}\right)^{2} \tanh \left(\frac{z e \Psi_{\mathrm{NP}}}{4 k_{\mathrm{B}} T}\right) \tanh \left(\frac{z e \Psi_{\mathrm{int}}}{4 k_{\mathrm{B}} T}\right)\left[(\kappa r-1) e^{-\kappa h}+(1+\kappa r) e^{-\kappa(h+2 r)}\right]
\end{aligned}
$$

In the equations Eq. 12 and $13 \varepsilon$ is the dielectric permittivity of the medium, $\kappa^{-1}$ is the Debye length $\Psi_{\mathrm{NP}}, \Psi_{\text {int }}$ and $\Psi_{\text {im }}$ are the surface potential of the particle, the interface and the image charge.

The interfacial surface potential of octane/water and octanol/water were estimated by measuring the zeta potential of their emulsions. $-32 \mathrm{mV}$ zeta potential was measured for the octane droplets and $-38 \mathrm{mV}$ for octanol. For the air-water interface a zeta potential value of $-15 \mathrm{mV}$ was used [47].

A critical factor in the calculations is the ionic strength of the medium affecting the value of the Debye length. As the particles were suspended in pure water the ionic strength of the samples originates from the presence of the nanoparticles themselves. Knowing the size and mass concentration of the particles and taking into consideration the molar mass of the PLGA polymers the number of surface carboxylic end groups can be estimated in a given volume. The calculations added up to $2 \times 10^{-5} \mathrm{M}$ surface carboxylic groups. This value corresponds well with the measured $4.5 \mathrm{pH}$ of the samples.

$U_{\text {tot }}$ and its components, the calculated interaction energies of a NP and a given interface at an example $5 \mathrm{~nm}$ separation distance are listed in Table 3. 
Table 3. Interaction energy and its components between a particle and interface at a fixed $5 \mathrm{~nm}$ separation distance.

\begin{tabular}{llcccc}
\hline Sample & Interface & $U_{\mathrm{vdw}} / \mathrm{k}_{\mathrm{B}} \mathrm{T}$ & $U_{\mathrm{EDL}} / \mathrm{k}_{\mathrm{B}} \mathrm{T}$ & $U_{\text {image }} / \mathrm{k}_{\mathrm{B}} \mathrm{T}$ & $U_{\text {tot }} / \mathrm{k}_{\mathrm{B}} \mathrm{T}$ \\
\hline PLGA & air/water & 3.2 & 7.9 & 58.3 & 69.4 \\
PLGA-Pluronic & air/water & 3.2 & 3.3 & 10.1 & 16.6 \\
PLGA & octane/water & -0.3 & 16.5 & 57.2 & 73.3 \\
PLGA-Pluronic & octane/water & -0.3 & 6.9 & 9.8 & 16.4 \\
PLGA & octanol/water & -1.3 & 19.3 & 48.4 & 66.4 \\
PLGA-Pluronic & octanol/water & -1.3 & 8.1 & 8.1 & 14.8 \\
\hline
\end{tabular}

Apart from very small separation distances where van der Waals interactions dominate (repulsive for air, attractive for octane and octanol), in all cases a long range repulsion exists between the interfaces and the particles that is mainly electrostatic in nature. Repulsive image charge interactions dominate particle interface interactions even at significant separation distances due to the low ionic strength medium. Pure PLGA particles experience a significantly higher energy barrier approaching the interface compared to Pluronic stabilized particles. This is due to the electrostatic screening effect of the Pluronic surface layer that reduces the zeta potential of these particles. On the other hand no significant differences could be observed in the calculated energy values when the three interfaces are compared. In all cases the energy barriers are much higher than the average kinetic energy of the particles therefore the adsorption process is significantly hindered as indicated by the small $D_{\text {eff }}$ values. As for Pluronic stabilizes particles $U_{\text {tot }}$ is six times smaller than the pure PLGA particles and could be a contributing factor to their increased rate of adsorption.

\section{Interfacial film structure}

PLGA and PLGA-Pluronic NPs exhibit significantly different surface pressure - area isotherms. The Pluronic coated particle film exerts a significantly larger surface pressure compared to PLGA NPs at identical smaller surface concentrations but become similar at high compression. This behavior can be attributed to the surface activity of the surface bound Pluronic molecules. It is a well known phenomenon that soft microgel particles and small nanoparticles with surface active covering layer are deformed at the interface to occupy the largest possible surface area [12,22]. At $25{ }^{\circ} \mathrm{C}$ PLGA is below the glass transition temperature therefore the particle cores can be considered rigid. On a belt around the three-phase contact line of the particles, Pluronic molecules can directly interact with the interface thereby reducing the surface tension. Previously the area occupied by a Pluronic molecule on a PLGA nanoparticle surface has been determined as around $20 \mathrm{~nm}^{2} /$ molecule [48]. Using this value the number of Pluronic molecules at the particle surface can be calculated. The number of Pluronic molecules that are able to interact directly with the interface was calculated by assuming varying thicknesses of the belt around the contact line. The effect of these Pluronic molecules on the surface pressure was determined from the surface pressure - area isotherm of Pluronic (Fig. 7). 


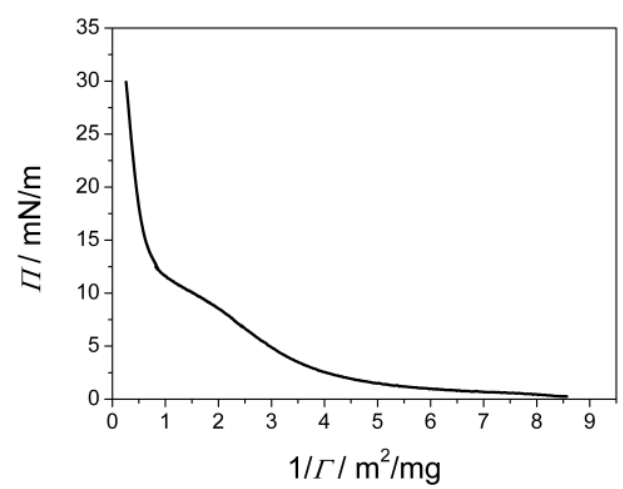

Figure 7. Surface pressure - area isotherm of Pluronic F127

It was found that assuming a $16 \mathrm{~nm}$ wide belt on the particles surrounding the contact lines the amount of Pluronic molecules residing in these areas would lower the surface tension by $15.2 \mathrm{mN} / \mathrm{m}$. If this value is subtracted from the equilibrium surface pressure of the Pluronic modified nanoparticles the resulting surface pressure is equal to the one determined for pure PLGA nanoparticles. This means that the surface tension lowering mechanism of the Pluronic coated particles seems to be additive and the particles behave as a reservoir for the polymer surfactant. The presence of Pluronic molecules at the interface is also indicated by the measured dilatational modulus values. At surface pressures in the range of 17 and $33 \mathrm{mN} / \mathrm{m}$ the value of $|E|$ is around $22 \mathrm{mN} / \mathrm{m}$, close to that of pure Pluronic $(18 \mathrm{mN} / \mathrm{m})$.

To get a better understanding of the interparticle interactions at the interface calculations were performed according to the DLVO theory, taking into account the van der Waals $\left(U_{\mathrm{vdW}}\right)$ and electric double layer $\left(U_{\mathrm{EDL}}\right)$ interactions and expanded by steric repulsion $\left(U_{\mathrm{St}}\right)$ interaction. The van der Waals interaction between two spheres at an interface can be expressed as

$U_{\mathrm{vdW}}=-\frac{A_{\text {int }}}{6}\left[\frac{2 r^{2}}{h^{2}+4 r h}+\frac{2 r^{2}}{(h+2 r)^{2}}+\ln \left(1-\frac{4 r^{2}}{(h+2 r)^{2}}\right)\right]$

where $r$ is the radius of the particle, $h$ is the separation distance. The interfacial Hamaker constant of the particle, $A_{\text {int }}$, can be estimated with the expression

$$
A_{\text {int }}=\left(A_{11}^{1 / 2}-A_{22}^{1 / 2}\right)^{2}+\left(\frac{1-\cos \theta}{2}\right)^{2}(2+\cos \theta)\left[\left(A_{11}^{1 / 2}-A_{33}^{1 / 2}\right)^{2}-\left(A_{11}^{1 / 2}-A_{22}^{1 / 2}\right)^{2}\right]
$$

where $\theta$ is the contact angle of the particle at the interface, $A_{11}, A_{22}$ and $A_{33}$ are the vacuum Hamaker constants of the particle, water and the other phase respectively [49].

The electric double layer interaction between the two particles was calculated based on the linear superposition approximation of the Poisson-Boltzmann equation as given by Bell et al. for two equivalent spheres:

$U_{\mathrm{EDL}}=32 \pi \varepsilon_{\mathrm{r}} \varepsilon_{0} r\left(\frac{k_{\mathrm{B}} T}{z e}\right)^{2} \tanh ^{2}\left(\frac{z e \Psi_{\mathrm{NP}}}{4 k_{\mathrm{B}} T}\right) e^{-\kappa h}$

where $\varepsilon$ is the dielectric permittivity of the medium, $\kappa^{-1}$ is the Debye length, $r$ and $\Psi_{\mathrm{NP}}$ are the radius and surface potential of the particle and $h$ is the separation distance.

The steric interaction can be approximated with the following formulae [50,51]: 


$$
\begin{aligned}
& U_{s t}=\tilde{\Gamma} k_{\mathrm{B}} T\left[\frac{\pi^{2} L^{2}}{3 h^{2}}-\ln \left(\frac{8 \pi L^{2}}{3 h^{2}}\right)\right] \mid h \leq L^{2} \sqrt{3} \\
& U_{s t}=4 \tilde{\Gamma} k_{\mathrm{B}} T \exp \left(-\frac{3 h^{2}}{2 L^{2}}\right) \mid h>L^{2} \sqrt{3}
\end{aligned}
$$

where $h$ is the separation distance, $L$ is the thickness of the brush that was estimated as $L \approx l N^{1 / 2}$, where $l$ is the length of a polymeric segment, $3.7 \AA$ for PEO [52,53], and $N$ is the number of segments, 258 for Pluronic F127. $\tilde{\Gamma}$ the dimensionless reduced surface coverage was obtained as

$$
\tilde{\Gamma}=\frac{\Gamma N v m}{N_{\mathrm{A}} l},
$$

where $\Gamma$ was calculated based on the molecular surface area of Pluronic on PLGA, $20 \mathrm{~nm}^{2}$ [48], $m$ is the average molar weight of a segment and $v$ is the average specific volume of a segment that was calculated from the data for PEO and PPO, 0.84 and $0.92 \mathrm{~cm}^{3} / \mathrm{g}$ respectively [54].

The total interaction energies for PLGA and PLGA-Pluronic nanoparticles are presented in Fig. 8.

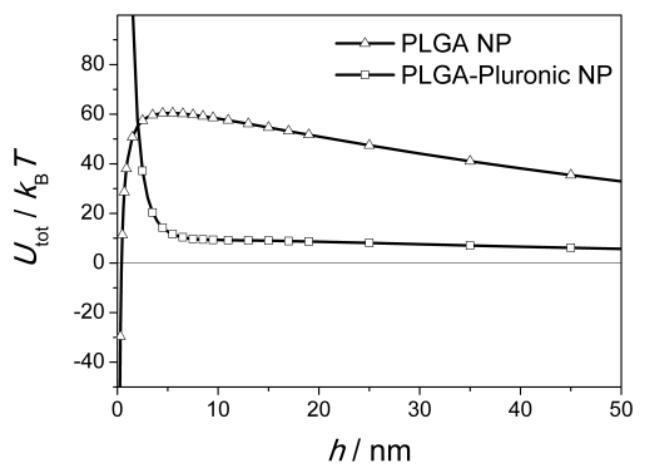

Figure 8. Interparticle interaction for PLGA and PLGA-Pluronic NPs at the air/water interface as a function of separation distance

In both cases a strong repulsion exists between the particles. Due to the low ionic strength environment electrostatic interactions dominate at long ranges. This long range repulsion appears to be lower in the case of Pluronic stabilized particles as a consequence of the screening effect of the polymer cover layer. However, even in this case the magnitude of the interaction is several times higher than the particles thermal energy. This behavior is reflected in the dilatational moduli of the films. At low surface concentrations pure PLGA particle films had almost two times greater $|E|$ values than the Pluronic coated NPs indicating higher long range repulsion between the particles. The stepwise increase in $|E|$ at increased compression for the PLGA-Pluronic particles marks the onset of interaction between the Pluronic brushes. When the particles are brought into closer contact the two systems behave differently. In the case of the pure PLGA particles at close distances the attractive van der Waals forces become dominant leading to aggregation. This effect is observable in the AFM images of pure PLGA particle films (Fig. 5). On the other hand a strong repulsive steric interaction arises between Pluronic stabilized particles at close contact that enables particles to remain kinetically independent even when pressed close together.

\section{Conclusions}

The surface activity of the PLGA and Pluronic modified PLGA NPs was evaluated at three fluid interfaces. Water/air, water/octane and water/octanol interfaces possessing decreasing interfacial tension provides a series approaching the polarity conditions valid at biological membrane surfaces. 
The accumulation of PLGA NPs at these interfaces can be adequately described by the calculated surface free energy change [36]. The surface activity of Pluronic modified PLGA NPs however, was found to be significantly higher than expected. That increased surface activity was evaluated as the result of contributions of both the PLGA NPs and the effective portion of their Pluronic shell. The presence of Pluronic at the NP surface also introduces strong repulsive steric interactions able to prevent the irreversible particle aggregation observed in highly compressed PLGA NP layer.

To describe the interfacial adsorption and its kinetics the diffusion controlled adsorption theory had to be completed by the consideration of the energy barrier of particle transport to the interface. This type of approach was applied for small silica nanoparticles previously [24]. We showed that, although considered a hydrophobic material, pure PLGA NPs bear significant surface charge and hence this system can be similarly treated. That surface charge is also sufficient to form interfacial layer with kinetically separate particles. Analysis of the adsorption kinetics showed that a large electrostatic energy barrier between the PLGA NPs and the interfaces slows down the adsorption process. The increased adsorption rate found for Pluronic covered PLGA NPs is the consequence of decreased electrostatic barrier present due to the polymer screening effect.

The surface free energy of adsorption calculated for the accumulation of particles at the three fluid interfaces suggested the preference of adsorption with the one exception of PLGA NPs at water/octanol interface. The surface modification of PLGA NPs by Pluronic led to notable surface activity even in that case. This finding can be related to the biological membrane affinity of PLGA NPs observed experimentally previously [48], where the Pluronic coating significantly increased the penetration of particles into lipid monolayers.

Such detailed analysis of the particle layer formation process is planned to extend to systems with complex core-shell structure and to the influence of parameters like temperature and ionic strength. The gained insights could help describing the interfacial properties of other polymer stabilized particle systems.

\section{Acknowledgements}

This work was supported by the National Science Foundation: OTKA 104928, OTKA NK 105898 and Hungarian Academy of Sciences Postdoctoral Research Program. The authors thank Á. Ábrahám, J. Orosz and B. Szabados for their contributions to the surface tension and Langmuir monolayer measurements.

\section{References}

[1] L. Nyström, M. Malmsten, Surface-bound microgels - From physicochemical properties to biomedical applications, Adv. Colloid Interfac. 238 (2016) 88-104.

DOI: $10.1016 / j . c i s .2016 .11 .003$

[2] Y. Lin, H. Skaff, A. Böker, A.D. Dinsmore, T. Emrick, T.P. Russell, Ultrathin Cross-Linked Nanoparticle Membranes, J. Am. Chem. Soc. 125 (2003) 12690-12691.

DOI: $10.1021 / \mathrm{ja} 036919 \mathrm{a}$

[3] C.P. Collier, R.J. Saykally, J.J. Shiang, S.E. Henrichs, J.R. Heath, Reversible Tuning of Silver Quantum Dot Monolayers Through the Metal-Insulator Transition, Science 277 (1997) 19781981.

DOI: $10.1126 /$ science. 277.5334 .1978 
[4] Á. Detrich, N. Nagy, M. Nyári, E. Albert, D. Zámbó, Z. Hórvölgyi, Nanostructured antireflective bilayers: Optical design and preparation, Mater. Chem. Phys. 145 (2014) 176-185.

DOI: 10.1016/j.matchemphys.2014.01.056

[5] F. Nan, J. Wu, F. Qi, Q. Fan, G. Ma, T. Ngai, Preparation of uniform-sized colloidosomes based on chitosan-coated alginate particles and its application for oral insulin delivery, J. Mater. Chem. B 2 (2014) 7403-7409. DOI: 10.1039/C4TB01259C

[6] D. Marku, M. Wahlgren, M. Rayner, M. Sjöö, A. Timgren, Characterization of starch Pickering emulsions for potential applications in topical formulations, Int. J. Pharm. 428 (2012) 1-7.

DOI: 10.1016/j.ijpharm.2012.01.031

[7] T. Yi, C. Liu, J. Zhang, F. Wang, J. Wang, J. Zhang, A new drug nanocrystal self-stabilized Pickering emulsion for oral delivery of silybin, Eur. J. Pharm. Sci. 96 (2017) 420-427.

DOI: $10.1016 /$ j.ejps.2016.08.047

[8] N. Babcsán, D. Leitlmeier, J. Banhart, Metal foams—-high temperature colloids: Part I. Ex situ analysis of metal foams, Colloid. Surface. A 261 (2005) 123-130.

DOI: 10.1016/j.colsurfa.2004.12.030

[9] O. Zech, M.E. Haase, D.G. Shchukin, T. Zemb, Froth flotation via microparticle stabilized foams, Colloid. Surface. A 413 (2012) 2-6.

DOI: $10.1016 /$ j.colsurfa.2012.04.024

[10] B.P. Binks, D. Yin, Pickering emulsions stabilized by hydrophilic nanoparticles: in situ surface modification by oil, Soft Matter 12 (2016) 6858-6867.

DOI: $10.1039 /$ C6SM01214K

[11] F. Pinaud, K. Geisel, P. Massé, B. Catargi, L. Isa, W. Richtering, V. Ravaine, V. Schmitt, Adsorption of microgels at an oil-water interface: correlation between packing and 2D elasticity, Soft Matter 10 (2014) 6963-6974.

DOI: $10.1039 / \mathrm{c} 4 \mathrm{sm} 00562 \mathrm{~g}$

[12] K. Geisel, L. Isa, W. Richtering, Unraveling the 3D Localization and Deformation of Responsive Microgels at Oil/Water Interfaces: A Step Forward in Understanding Soft Emulsion Stabilizers, Langmuir 28 (2012) 15770-15776.

DOI: $10.1021 / \mathrm{la} 302974 \mathrm{j}$

[13] M. Destribats, M. Eyharts, V. Lapeyre, E. Sellier, I. Varga, V. Ravaine, V. Schmitt, Impact of pNIPAM Microgel Size on Its Ability To Stabilize Pickering Emulsions, Langmuir 30 (2014) 1768-1777.

DOI: $10.1021 / \mathrm{la} 4044396$

[14] F. Qi, J. Wu, G. Sun, F. Nan, T. Ngai, G. Ma, Systematic studies of Pickering emulsions stabilized by uniform-sized PLGA particles: preparation and stabilization mechanism, J. Mater. Chem. B 2 (2014) 7605-7611.

DOI: $10.1039 / \mathrm{c} 4 \mathrm{tb} 01165 \mathrm{a}$ 
[15] M.J. Santander-Ortega, A.B. Jódar-Reyes, N. Csaba, D. Bastos-González, J.L. Ortega-Vinuesa, Colloidal stability of Pluronic F68-coated PLGA nanoparticles: a variety of stabilisation mechanisms, J. Colloid Interf. Sci. 302 (2006) 522-529.

DOI: $10.1016 /$ j.jcis.2006.07.031

[16] W. Chaisri, W.E. Hennink, S. Okonogi, Preparation and characterization of cephalexin loaded PLGA microspheres, Curr. Drug. Deliv. 6 (2009) 69-75.

DOI: $10.2174 / 156720109787048186$

[17] É. Kiss, D. Schnöller, K. Pribranská, K. Hill, C.B. Pénzes, K. Horváti, S. Bősze,

Nanoencapsulation of antitubercular drug isoniazid and its lipopeptide conjugate, J. Disper. Sci. Technol. 32 (2011) 1728-1734.

DOI: $10.1080 / 01932691.2011 .616128$

[18] É. Kiss, E. Kutnyánszky, I. Bertóti, Modification of Poly(lactic/glycolic acid) Surface by Chemical Attachment of Poly(ethylene glycol), Langmuir 26 (2010) 1440-1444.

DOI: $10.1021 / \mathrm{la} 903373 \mathrm{~g}$

[19] G. Gyulai, C.B. Pénzes, M. Mohai, T. Lohner, P. Petrik, S. Kurunczi, É. Kiss, Interfacial properties of hydrophilized poly(lactic-co-glycolic acid) layers with various thicknesses, J. Colloid Interf. Sci. 362 (2011) 600-606.

DOI: 10.1016/j.jcis.2011.06.055

[20] É. Kiss, M.G. Takács, I. Bertóti, E.I. Vargha-Butler, Surface properties of poly(lactic/glycolic acid)-pluronic ${ }^{\circledR}$ blend films, Polym. Advan. Technol. 14 (2003) 839-846.

DOI: $10.1002 /$ pat.404

[21] M. Malmsten. Protein adsorption in intravenous drug delivery. In: M. Malmsten, ed. Biopolymers at interfaces. Marcel Dekker; 1998.

[22] R.W. Murphy, B.E. Farkas, O.G. Jones, Dynamic and viscoelastic interfacial behavior of $\beta$ lactoglobulin microgels of varying sizes at fluid interfaces, J. Colloid Interf. Sci. 466 (2016) 1219.

DOI: $10.1016 /$ j.jcis.2015.12.012

[23] A.G. Bykov, B.A. Noskov, G. Loglio, V.V. Lyadinskaya, R. Miller, Dilational surface elasticity of spread monolayers of polystyrene microparticles, Soft Matter 10 (2014) 6499-6505.

DOI: $10.1039 / \mathrm{c} 4 \mathrm{sm} 00782 \mathrm{~d}$

[24] V.R. Dugyala, J.S. Muthukuru, E. Mani, M.G. Basavaraj, Role of electrostatic interactions in the adsorption kinetics of nanoparticles at fluid-fluid interfaces, Phys. Chem. Chem. Phys. 18 (2016) 5499-5508.

DOI: $10.1039 / \mathrm{c} 5 \mathrm{cp} 05959 \mathrm{c}$

[25] T. Kobayashi, M. Kawaguchi, Surface dilational moduli of latex-particle monolayers spread at air-water interface, J. Colloid Interf. Sci. 390 (2013) 147-150.

DOI: $10.1016 /$ j.jcis.2012.09.050 
[26] G. Gyulai, C.B. Pénzes, M. Mohai, F. Csempesz, É. Kiss, Influence of surface properties of polymeric drug delivery nanoparticles on their membrane affinity, Eur. Polym. J. 49 (2013) 24952503.

DOI: $10.1016 /$ j.eurpolymj.2013.02.024

[27] G. Gyulai, A. Magyar, J. Rohonczy, J. Orosz, M. Yamasaki, S. Bősze, É. Kiss, Preparation and characterization of cationic Pluronic for surface modification and functionalization of polymeric drug delivery nanoparticles, Express Polym. Lett. 10 (2016) 216-226.

DOI: 10.3144/expresspolymlett.2016.20

[28] K.G. Marinova, R.G. Alargova, N.D. Denkov, O.D. Velev, D.N. Petsev, I.B. Ivanov, R.P. Borwankar, Charging of Oil-Water Interfaces Due to Spontaneous Adsorption of Hydroxyl Ions, Langmuir 12 (1996) 2045-2051.

DOI: $10.1021 / \mathrm{la} 950928 \mathrm{i}$

[29] É. Kiss, E.T. Heine, K. Hill, Y.C. He, N. Keusgen, C.B. Pénzes, D. Schnöller, G. Gyulai, A. Mendrek, H. Keul, et al., Membrane affinity and antimicrobal properties of polyelectrolytes with different hydrophobicity, Macromol. Biosci. 12 (2012) 1181-1189.

[30] R. Hegedüs, M. Manea, E. Orbán, I. Szabó, É. Kiss, É. Sipos, G. Halmos, G. Mező, Enhanced cellular uptake and in vitro antitumor activity of short-chain fatty acid acylated daunorubicinGnRH-III bioconjugates, Eur. J. Med. Chem. 56 (2012) 155-165.

DOI: 10.1016/j.ejmech.2012.08.014

[31] F. Ravera, G. Loglio, V.I. Kovalchuk, Interfacial dilational rheology by oscillating bubble/drop methods, Curr. Opin. Colloid In. 15 (2010) 217-228.

DOI:10.1016/j.cocis.2010.04.001

[32] É. Kiss, I. Bertóti, E.I. Vargha-Butler, XPS and Wettability Characterization of Modified Poly(lactic acid) and Poly(lactic/glycolic acid) Films, J. Colloid Interf. Sci. 245 (2002) 91-98. DOI: $10.1006 /$ jcis.2001.7954

[33] V.B. Fainerman, A.V. Makievski, R. Miller, The analysis of dynamic surface tension of sodium alkyl sulphate solutions, based on asymptotic equations of adsorption kinetic theory, Colloid. Surface. A 87 (1994) 61-75. DOI: $10.1016 / 0927-7757(94) 02747-1$

[34] N.J. Alvarez, S.L. Anna, T. Saigal, R.D. Tilton and L.M. Walker, Interfacial Dynamics and Rheology of Polymer-Grafted Nanoparticles at Air-Water and Xylene-Water Interfaces, 28 (2012) 8052-8063.

DOI: $10.1021 / \mathrm{la} 300737 \mathrm{p}$

[35] S. Razavi, K.D. Cao, B. Lin, K.Y.C. Lee, R.S. Tu, I. Kretzschmar, Collapse of Particle-Laden Interfaces under Compression: Buckling vs Particle Expulsion, Langmuir 31 (2015) 7764-7775. DOI: 10.1021/acs.langmuir.5b01652 
[36] Z. Hórvölgyi, S. Németh, J.H. Fendler, Monoparticulate Layers of Silanized Glass Spheres at the Water-Air Interface: Particle-Particle and Particle-Subphase Interactions, Langmuir 12 (1996) 997-1004.

DOI: $10.1021 / 1 \mathrm{la} 9406580$

[37] A.F.H. Ward, L. Tordai, Time-Dependence of Boundary Tensions of Solutions I. The Role of Diffusion in Time-Effects, J. Chem. Phys. 14 (1946) 453-461.

DOI: $10.1063 / 1.1724167$

[38] N. Bizmark, M.A. Ioannidis, D.E. Henneke, Irreversible Adsorption-Driven Assembly of Nanoparticles at Fluid Interfaces Revealed by a Dynamic Surface Tension Probe, Langmuir 30 (2014) $710-717$.

DOI: $10.1021 / \mathrm{la} 404357 \mathrm{j}$

[39] S. Bhattacharjee, M. Elimelech, Surface Element Integration: A Novel Technique for Evaluation of DLVO Interaction between a Particle and a Flat Plate, J. Colloid Interf. Sci. 193 (1997) 273285.

DOI: $10.1006 /$ jcis. 1997.5076

[40] J. Chen, M.K. Lee, E. Qin, S. Misrab, H. Kong, van der Waals force-induced loading of proangiogenic nanoparticles on microbubbles for enhanced neovascularization, Nanoscale 7 (2015) 17139-17147.

DOI: $10.1039 / C 5 N R 03399 C$

[41] R.J. Hunter. Foundations of Colloid Science. Oxford University Press; 2001.

[42] P.C. Hiemenz, R. Rajagopalan. Principles of Colloid and Surface Chemistry. Marcel Dekker, Inc; 1997.

[43] W.C. Duncan-Hewitt, Oriented Dipoles at Interfaces: Calculation of Surface Potential and Surface Tension, Langmuir 7 (1991) 1229-1234.

DOI: $10.1021 / \mathrm{la} 00054 \mathrm{a} 035$

[44] H. Wang, V. Singh, S.H. Behrens, Image Charge Effects on the Formation of Pickering Emulsions, J. Phys. Chem. Lett. 3 (2012) 2986-2990.

DOI: $10.1021 / \mathrm{jz} 300909 \mathrm{z}$

[45] J.N. Israelachvili. Intermolecular and Surface Forces. Third Edition ed. Academic Press; 2011.

[46] S. Lin, M.R. Wiesner, Exact Analytical Expressions for the Potential of Electrical Double Layer Interactions for a Sphere-Plate System, Langmuir 26 (2010) 16638-16641.

DOI: $10.1021 / \mathrm{la} 103046 \mathrm{w}$

[47] S. Cho, J. Kim, J. Chun, J. Kim, Ultrasonic formation of nanobubbles and their zeta-potentials in aqueous electrolyte and surfactant solutions, Colloid. Surface. A 269 (2005) 28-34.

DOI: $10.1016 /$ j.colsurfa.2005.06.063 
[48] É. Kiss, G. Gyulai, C.B. Pénzes, M. Idei, K. Horváti, B. Bacsa, S. Bősze, Tuneable surface modification of PLGA nanoparticles carrying new antitubercular drug candidate, Colloid. Surface. A 458 (2014) 178-186.

DOI: 10.1016/j.colsurfa.2014.05.048

[49] V. Garbin, J.C. Crocker, K.J. Stebe, Nanoparticles at fluid interfaces: Exploiting capping ligands to control adsorption, stability and dynamics, J. Colloid Interf. Sci. 387 (2012) 1-11.

DOI: $10.1016 /$ j.jcis.2012.07.047

[50] A.K. Dolan, S.F. Edwards, The effect of excluded volume on polymer dispersant action, Proc. R. Soc. Lond. A 343 (1975) 427-442.

DOI: 10.1098/rspa.1975.0077

[51] P. Kralchevsky, K. Nagayama. Particles at fluid interfaces and membranes. New York: Elsevier Science; 2001.

[52] H. Lee, R.M. Venable, A.D. MacKerell, R.W. Pastor, Molecular Dynamics Studies of Polyethylene Oxide and Polyethylene Glycol: Hydrodynamic Radius and Shape Anisotropy, Biophys. J. 95 (2008) 1590-1599.

DOI: 10.1529/biophysj.108.133025

[53] É. Kiss, K. Dravetzky, K. Hill, E. Kutnyánszky, A. Varga, Protein interaction with a Pluronicmodified poly(lactic acid) Langmuir monolayer, J. Colloid Interf. Sci. 325 (2008) 337-345. DOI:10.1016/j.jcis.2008.05.057

[54] C. Sommer, J.S. Pedersen, P.C. Stein, Apparent Specific Volume Measurements of Poly(ethylene oxide), Poly(butylene oxide), Poly(propylene oxide), and Octadecyl Chains in the Micellar State as a Function of Temperature, J. Phys. Chem. B 108 (2004) 6242-6249.

DOI: $10.1021 / \mathrm{jp} 036963 \mathrm{c}$ 
Figure captions

Figure 1. Experimental setup of the captive bubble/droplet contact angle measurement and its relation to the particle contact angle at the interface

Figure 2. Surface and interfacial tension of PLGA and Pluronic modified PLGA NPs as a function of time at concentration of $2 \mathrm{~g} / \mathrm{L}$, and equilibrium surface and interfacial tension values as a function of initial bulk concentration

Figure 3. Surface pressure - area isotherm of PLGA and PLGA-Pluronic NPs at the air/water interface

Figure 4. Dilatational modulus $(|E|)$ and its components $\left(E^{\prime}, E^{\prime \prime}\right)$ of PLGA and PLGA-Pluronic NPs at the air/water interface as a function of surface pressure

Figure 5. AFM image of PLGA and PLGA-Pluronic NP layers transferred at $12 \mathrm{mN} / \mathrm{m}$ (A: PLGA, C: PLGA-Pluronic) and $30 \mathrm{mN} / \mathrm{m}$ (B: PLGA, D: PLGA-Pluronic)

Figure 6. Interfacial tension of nanoparticles at the octane/water interface as a function of the square root of time

Figure 7. Surface pressure - area isotherm of Pluronic F127

Figure 8. Interparticle interaction for PLGA and PLGA-Pluronic NPs at the air/water interface as a function of separation distance 
Tables

Table 1. Equilibrium surface pressure $\left(\Pi_{\mathrm{eq}}\right)$ and dilatational modulus $(|E|)$ of nanoparticle layer at different interfaces

\begin{tabular}{|c|c|c|c|c|c|}
\hline \multirow[t]{2}{*}{ Interface } & \multirow{2}{*}{$\begin{array}{c}\text { Pure interface } \\
\gamma_{0} / \mathrm{mN} / \mathrm{m}\end{array}$} & \multicolumn{2}{|c|}{ PLGA NP } & \multicolumn{2}{|c|}{ PLGA-Pluronic NP } \\
\hline & & $\Pi_{\mathrm{eq}} / \mathrm{mN} / \mathrm{m}$ & $|E| / \mathrm{mN} / \mathrm{m}$ & $\Pi_{\mathrm{eq}} / \mathrm{mN} / \mathrm{m}$ & $|E| / \mathrm{mN} / \mathrm{m}$ \\
\hline air/water & 72.0 & 16.0 & 9.8 & 31.2 & 10.0 \\
\hline n-octane/water & 50.0 & 20.2 & 10.6 & 41.0 & 14.6 \\
\hline 1-octanol/water & 8.5 & 0.1 & 0.1 & 2.1 & 1.7 \\
\hline
\end{tabular}

Table 2. Contact angle $(\theta)$, calculated free energy of adsorption $(\Delta G)$, Stokes-Einstein $\left(D_{0}\right)$ and effective $\left(D_{\text {eff }}\right)$ diffusion coefficient of NPs at different interfaces

\begin{tabular}{llcccc}
\hline Particle & Interface & $\theta /{ }^{\circ}$ & $\Delta G / k_{\mathrm{B}} T$ & $D_{0} / \mathrm{m}^{2} / \mathrm{s}$ & $D_{\text {eff }} / \mathrm{m}^{2} / \mathrm{s}$ \\
\hline PLGA & air/water & 74 & $-5.2 \times 10^{4}$ & $5.8 \times 10^{-12}$ & $1.0 \times 10^{-14}$ \\
PLGA-Pluronic & air/water & 65 & $-3.9 \times 10^{4}$ & $5.3 \times 10^{-12}$ & $4.0 \times 10^{-14}$ \\
PLGA & n-octane/water & 100 & $-4.7 \times 10^{4}$ & $5.8 \times 10^{-12}$ & $2.2 \times 10^{-14}$ \\
PLGA-Pluronic & n-octane/water & 106 & $-4.2 \times 10^{4}$ & $5.3 \times 10^{-12}$ & $1.6 \times 10^{-13}$ \\
PLGA & 1-octanol/water & 163 & $-2.1 \times 10^{1}$ & $5.8 \times 10^{-12}$ & $2.3 \times 10^{-14}$ \\
PLGA-Pluronic & 1-octanol/water & 143 & $-5.6 \times 10^{2}$ & $5.3 \times 10^{-12}$ & $7.4 \times 10^{-14}$
\end{tabular}

*Note: Contact angle values were reproducible to $\pm l^{\circ}$ leading to an error of $\pm 10 \%$ in the calculated $\Delta G$ values

Table 3. Interaction energy and its components between a particle and interface at a fixed $5 \mathrm{~nm}$ separation distance.

\begin{tabular}{llcccc}
\hline Sample & Interface & $U_{\mathrm{vdw}} / \mathrm{k}_{\mathrm{B}} \mathrm{T}$ & $U_{\mathrm{EDL}} / \mathrm{k}_{\mathrm{B}} \mathrm{T}$ & $U_{\text {image }} / \mathrm{k}_{\mathrm{B}} \mathrm{T}$ & $U_{\text {tot }} / \mathrm{k}_{\mathrm{B}} \mathrm{T}$ \\
\hline PLGA & air/water & 3.2 & 7.9 & 58.3 & 69.4 \\
PLGA-Pluronic & air/water & 3.2 & 3.3 & 10.1 & 16.6 \\
PLGA & octane/water & -0.3 & 16.5 & 57.2 & 73.3 \\
PLGA-Pluronic & octane/water & -0.3 & 6.9 & 9.8 & 16.4 \\
PLGA & octanol/water & -1.3 & 19.3 & 48.4 & 66.4 \\
PLGA-Pluronic & octanol/water & -1.3 & 8.1 & 8.1 & 14.8 \\
\hline
\end{tabular}

